Noname manuscript No.

(will be inserted by the editor)

\title{
A Taylor series based continuation method for solutions of dynamical systems
}

\author{
Louis Guillot (corresponding author) • Bruno Cochelin • \\ Christophe Vergez
}

Received: date / Accepted: date

\begin{abstract}
This paper describes a generic Taylor series based continuation method, the so-called Asymptotic Numerical Method, to compute the bifurcation diagrams of nonlinear systems. The key point of this approach is the quadratic recast of the equations as it allows to treat in the same way a wide range of dynamical systems and their solutions. Implicit Differential-Algebraic Equations, forced or autonomous, possibly with time-delay or fractional order derivatives are handled in the same framework. The static, periodic and quasi-periodic solutions can be continued as well as transient solutions.
\end{abstract}

Keywords Nonlinear dynamics · Numerical continuation · Quadratic recast · Asymptotic Numerical Method · Taylor series · Dynamical systems.

\section{Introduction}

Numerical continuation methods are commonly used in different scientific communities. In this paper, one Taylor series based continuation method, also called the Asymptotic Numerical Method (ANM), is applied to various solutions of different types of dynamical systems. When the dynamical system of interest is an Ordinary Differential Equations (ODE) system, there are a lot of methods available in the literature. The continuation of equilibrium is a common possibility of $[9,16,27]$. The detection and the continuation of bifurcation points has also been investigated in $[13,14,28]$. These tools often allow the continuation of periodic orbits of ODE and of their bifurcations. More recently, some numerical tools were generalized to the continuation of equilibrium and periodic orbits of Delay Differential Equations [17] and of neutral type DDE [1]. The methods developed for the representation of periodic solutions can be time-domain methods e.g piecewise polynomial orthogonal collocation [15] or shooting [38,40], mixed time and frequency methods e.g trigonometric collocation [25], alternating frequency-time methods [5,18], and frequency domain method with the full harmonic balance method $[29,36]$. In the last few years, progress has been achieved on the continuation of quasi-periodic solutions of ODE, using different methods. In [39] the invariant tori are continued when in [19,43], a two dimensional alternating frequency-time scheme is developed. In [24], the system of equations is recast quadratically and a full quasi-periodic harmonic balance method is applied. Most of the methods presented above are designed for steady states regimes. However, they generally include the possibility of continuing solutions of boundary value problems and initial value problems if they are formulated as solutions of algebraic systems.

Commonly, the continuation tools use predictor-corrector methods for which the solutions on the branch are computed from (a) previous solution(s) and a predictor method, generally of first order. Then, a corrector is applied to the predicted solution to ensure that the corrected solution belongs to the solution branch. On the contrary, in this paper the continuation method is the Asymptotic Numerical Method (ANM) which is based on a high-order Taylor series expansion of the solution branch. It can be seen as a high-order predictor method which most of the time does not require a correction step. In a way, this is similar to homotopy techniques which are presented in [34]. The representation as a Taylor series gives a continuous representation of the solution branch, as opposed to predictor-corrector methods. Three main approaches to compute the

Louis Guillot (corresponding author), Christophe Vergez, Bruno Cochelin,

Aix Marseille Univ, CNRS, Centrale Marseille, LMA, UMR 7031,

4 impasse Nikola Tesla, 13013 Marseille, France.

E-mail: guillot@lma.cnrs-mrs.fr, vergez@lma.cnrs-mrs.fr,

bruno.cochelin@centrale-marseille.fr

Phone number : +33484524202 
Taylor series coefficients of the solution branch can be considered. The first one is to do the calculation by hand for a specific set of equations [4,8]. The second one uses automatic differentiation tools as in $[6,7]$. The third one relies on the quadratic recast of the equations. This latest approach is the one developed by the authors for its genericity and its performance [22]. It has first been described in $[9,10,11]$ in the case of equilibrium branches of structures and then extended to periodic solutions of ODE using the Harmonic Balance Method [12]. The generalization to the computation of quasi-periodic orbits has been done in [24]. The types of dynamical systems that can be treated in this framework is limited only by the need of a quadratic recast of the equations. This is far less restrictive than it could seem at first sight as will be shown throughout this paper. Periodic solutions of fractional order systems can be continued in this framework [42]. Some very recent work [22] has shown that the periodic solutions of delay differential equations (possibly of neutral type) could also be investigated with this method.

This article generalizes the approach given in [12]. The first section briefly recalls the content of [12] and gives the generalization to a large class of systems and to a large class of their solutions. The remaining sections treat some examples of applications in detail. For clear brevity reasons, the examples treated do not cover all the combinations of solution type and system type. In the second section, the solutions sought for are all timeperiodic. The periodic solutions of five types of dynamical systems, namely Ordinary Differential Equations (ODE), Differential Algebraic Equations (DAE), Implicit Differential Equations (IDE), Delay Differential Equations (DDE) and fractional order equations are investigated. It shows the versatility of our method in terms of dynamical system type. In the third section, a system of ODE is fixed. Its equilibrium, its periodic solutions, its quasi-periodic solutions and some of its transient regimes are continued. It shows the versatility of our method in terms of solution type.

\section{Elements of theory}

1.1 The quadratic framework and the Asymptotic Numerical Method

This section consists on a brief recall of the quadratic framework that is used by the continuation method developed by the authors. This is a key idea that allows a low time computation, an automatic and exact Jacobian matrix of the nonlinear system considered and a generic implementation which minimizes problem dependent implementation.

The so called Asymptotic Numerical Method (ANM) first described in $[9,10,11]$ is based on the numerical continuation of algebraic systems of the form

$$
\mathbf{R}(\mathbf{u}, \lambda)=0
$$

where $\mathbf{u} \in \mathbb{R}^{n}, \lambda \in \mathbb{R}$ is the continuation parameter and $\mathbf{R}: \mathbb{R}^{n} \times \mathbb{R} \mapsto \mathbb{R}^{n}$ is an analytic function of its arguments. From now on, the vector of all the unknowns $\mathbf{U}=(\mathbf{u}, \lambda)$ is introduced to simplify the notations. Let $\mathbf{U}_{0}$ be a regular solution ${ }^{1}$ of the system (1). Let $\mathbf{U}_{1}$ be a tangent vector at $\mathbf{U}_{0}$. The classical arc-length parameter [27] $a=\mathbf{U}_{1}^{t}\left(\mathbf{U}_{0}-\mathbf{U}\right)$ is introduced and the solution branch around $\mathbf{U}=\mathbf{U}_{0}$ is written as a power series $^{2}$ with respect to $a$

$$
\mathbf{U}(a)=\mathbf{U}_{0}+a \mathbf{U}_{1}+a^{2} \mathbf{U}_{2}+a^{3} \mathbf{U}_{3}+\cdots+a^{N} \mathbf{U}_{N} .
$$

This writing of the solution branch as a high-order power series is the base of the ANM. Several methods have been developed to compute the coefficients $\mathbf{U}_{p}, p \geq 1$ of the series. The approach presented in this paper consists on a quadratic recast of the equations (1) with the use of auxiliary variables $\mathbf{U}_{\mathrm{a}}$ :

$$
\mathbf{R}_{\mathrm{f}}\left(\mathbf{U}, \mathbf{U}_{\mathrm{a}}\right)=\mathbf{R}_{\mathrm{f}}\left(\mathbf{U}_{\mathrm{f}}\right)=\mathbf{C}+\mathbf{L}\left(\mathbf{U}_{\mathrm{f}}\right)+\mathbf{Q}\left(\mathbf{U}_{\mathrm{f}}, \mathbf{U}_{\mathrm{f}}\right)=0
$$

where $\mathbf{U}_{\mathrm{f}} \in \mathbb{R}^{\mathrm{n}_{\mathrm{f}}+1}, \mathrm{n}_{\mathrm{f}} \geq n$ and $\mathbf{C}, \mathbf{L}$ and $\mathbf{Q}$ are respectively a constant, a linear and a quadratic operator with values in $\mathbb{R}^{\mathrm{n}_{\mathrm{f}}}$. The subscript $f$ stands for full and the subscript $a$ stands for auxiliary. Once this recast is given, the auxiliary variables $\mathbf{U}_{\mathrm{a}}$ are expanded in Taylor series (2) in the same manner as for $\mathbf{U}$. The expansions are then introduced in the quadratic equations (3) and the terms of same order in $a$ are collected. It results in $N$ linear systems of size $\mathrm{n}_{\mathrm{f}}$ to be solved (sharing the same matrix to be inverted). The systems can be solved with the help of an efficient condensation algorithm which finally result in the Taylor series (2) sought for. The interested reader is referred to [22] for all the details on the practical computation of the series. It shall be emphasized here that once the quadratic recast is available, all the operations necessary

\footnotetext{
1 Regular means that the Jacobian matrix $\mathbf{J}$ at $\mathbf{U}_{0}$ is of full rank.

2 This is possible thanks to the analytic version of the implicit function theorem [20].
} 
to compute the Taylor series developments (2) can be automatized. Several examples of quadratic recasts are given throughout the paper. The next two subsections 1.1.1 and 1.1.2 give a hint of how generic this framework is through two examples. Firstly, it is shown how to recast polynomials and rational fractions quadratically. Secondly, a general method for the quadratic recast of transcendental functions is given. In the appendix C, the recurrence relations to compute the Taylor series development for these two examples are detailed.

\subsubsection{Rational nonlinearity}

The first step is to be able to recast rational fractions quadratically. To show the general idea of the method, the variable $y=\frac{1}{x}+x^{3}$ is recast quadratically. For this purpose, two auxiliary variables $u=\frac{1}{x}$ and $v=x^{2}$ are introduced. The second is already quadratic while the first one can be written implicitly $u x=1$. The case $x=0$ is not an issue here as this value is not a solution of the equation $u x=1$. The final quadratic recast of the variable $y$ is finally

$$
\left\{\begin{aligned}
y-u-x v & =0 \\
u x-1 & =0 \\
v-x^{2} & =0
\end{aligned}\right.
$$

This procedure can easily be generalized to any rational fraction. The appendix $\mathrm{C}$ details the computation of the coefficients of the series (2) for this example.

\subsubsection{Transcendental nonlinearity}

The next fundamental step is to be able to recast functions like exp, $\cos , \sin , \arctan , \ldots$ quadratically. The key is to use a differentiated form of these variables. In the book [21], these recasts are discussed in more details and a table of recasts of common functions is given in the article [22]. Hereafter, the example of sine function's recast is given as an highlight of the general approach.

Let us define the variable $y=\sin (x)$. Its differentiated form is $\mathrm{d} y=\cos (x) \mathrm{d} x$. An auxiliary variable $z=\cos (x)$, called companion variable, is introduced with its differentiated form $\mathrm{d} z=-\sin (x) \mathrm{d} x$. The final recast is

$$
\begin{cases}y-\sin (x)=0 \text { and } & \mathrm{d} y-z \mathrm{~d} x=0 \\ z-\cos (x)=0 \text { and } & \mathrm{d} z+y \mathrm{~d} x=0\end{cases}
$$

The original (not differentiated form) is used to compute the zero-th order coefficient of the series (2) and the differentiated form is used to compute higher order coefficients. This works easily as the differentiated form is quadratic with respect to the variables $(x, y, z, \mathrm{~d} x, \mathrm{~d} y, \mathrm{~d} z)$. This form is very generic and can be computed for all the elementary transcendental functions [21,22]. It gives the possibility to recast a very wide range of nonlinearities quadratically. The appendix $\mathrm{C}$ details the computation of the coefficients of the series (2) for this example.

\subsection{The classical ODE framework for periodic solution by HBM and ANM}

In [12], the case of periodic solutions of Ordinary Differential Equations (ODE) was treated. Let us consider the following ODE system :

$$
\dot{\mathbf{y}}=f(t, \mathbf{y}, \lambda)
$$

with $\mathbf{y}$ an unknown function of the time and $\lambda$ the continuation parameter. $f$ is an analytic function of its arguments and is time-periodic. As in [12], the unknown function $\mathbf{y}$ is expanded in truncated Fourier series :

$$
\mathbf{y}(t)=\mathbf{Y}_{0}+\sum_{k=1}^{H} \mathbf{Y}_{c, k} \cos (k \omega t)+\sum_{k=1}^{H} \mathbf{Y}_{s, k} \sin (k \omega t)
$$

The Harmonic Balance Method (HBM) consists in introducing this ansatz of $\mathbf{y}$ in the equations (6) and to balance the $2 H+1$ harmonic terms corresponding to $\mathbf{y}$ 's Fourier development. This yields an algebraic system of $2 H+1$ vectorial equations for $2 H+1$ vectorial unknowns $\mathbf{Y}_{0}, \mathbf{Y}_{c, k}, \mathbf{Y}_{s, k}$. The angular frequency $\omega$ is an additional unknown which is determined either by the angular frequency of the forcing term if the system is forced or by a phase condition [40] if the system is autonomous. 
A key point is that the algebraic system obtained after balancing the harmonics is automatically quadratic if the original system (6) has been recast quadratically before applying the HBM. Therefore, in the same manner as in the previous section and as explained in [12], auxiliary variables $\mathbf{y}_{\mathbf{a}}$ are introduced to obtain from (6) a system of the form

$$
\mathbf{d}\left(\dot{\mathbf{y}}_{\mathrm{f}}\right)=\mathbf{c}_{0}(t)+\lambda \mathbf{c}_{1}(t)+\mathbf{l}_{0}\left(\mathbf{y}_{\mathrm{f}}\right)+\lambda \mathbf{l}_{1}\left(\mathbf{y}_{\mathrm{f}}\right)+\mathbf{q}\left(\mathbf{y}_{\mathrm{f}}, \mathbf{y}_{\mathrm{f}}\right)
$$

where $\mathbf{y}_{\mathrm{f}}=\left(\mathbf{y}, \mathbf{y}_{\mathrm{a}}\right)$ is the full vector of unknowns and $\mathbf{c}_{0}, \mathbf{c}_{1}, \mathbf{l}_{0}, \mathbf{l}_{1}, \mathbf{d}, \mathbf{q}$ are respectively two constant operators, three linear operators and one bilinear operator. The form of equation (8) has been chosen to take into account that the continuation parameter $\lambda$ is not intended to be developed in Fourier series. In the reference [12] it is shown how to recover the constant, linear and bilinear operators of the algebraic system obtained after the harmonic balance from the operators of the system (8). It essentially gives the following algebraic system :

$$
\omega \mathbf{D}\left(\mathbf{Y}_{\mathrm{f}}\right)=\mathbf{C}_{0}+\lambda \mathbf{C}_{1}+\mathbf{L}_{0}\left(\mathbf{Y}_{\mathrm{f}}\right)+\lambda \mathbf{L}_{1}\left(\mathbf{Y}_{\mathrm{f}}\right)+\mathbf{Q}\left(\mathbf{Y}_{\mathrm{f}}, \mathbf{Y}_{\mathrm{f}}\right)
$$

where the capital letters stand for frequency domain operators and vector of Fourier coefficients. It means that, once the equations (6) has been recast in the form (8), its periodic solutions can be continued with the ANM as explained in section 1.1.

1.3 Generalization of the quadratic recast of ODE to a large class of dynamical systems and the continuation of their solution using ANM

Let us consider a differential system of the general form :

$$
f\left(t, \mathbf{y}, \dot{\mathbf{y}}, \ddot{\mathbf{y}}, \mathbf{y}(t-\tau), D^{\alpha}(\mathbf{y}), \lambda\right)=0
$$

where $\mathbf{y}$ is an unknown function of the time, $D^{\alpha}$ is the Weyl fractional order $\alpha$ derivative [42] operator and $f$ is an analytic function of its arguments which is time-periodic. The main objective of this paper is to show that a quadratic recast of this equation is possible and to show how this allows many types of solutions to be continued (equilibrium, periodic or quasi-periodic, transients) within a unique framework.

The first step is to introduce two auxiliary vectors $\mathbf{y}_{\mathrm{a}}^{\tau}(t)=\mathbf{y}(t-\tau)$ and $\mathbf{y}_{\mathrm{a}}^{\alpha}=D^{\alpha}(\mathbf{y})$ in order to isolate the time delays and the fractional order derivatives. These two terms are rewritten quadratically following the frequency-domain implementation developed in [42] and [23] respectively. The system (10) can now be written

$$
\begin{array}{r}
f\left(t, \mathbf{y}, \dot{\mathbf{y}}, \ddot{\mathbf{y}}, \mathbf{y}_{\mathrm{a}}^{\tau}, \mathbf{y}_{\mathrm{a}}^{\alpha}, \lambda\right)=0 \\
\mathbf{y}_{\mathrm{a}}^{\tau}(t)-\mathbf{y}(t-\tau)=0 \\
\mathbf{y}_{\mathrm{a}}^{\alpha}-D^{\alpha}(\mathbf{y})=0
\end{array}
$$

The two last equations are treated separately using appropriate methods (see [42] and [23]). The first equations are a set of Implicit Differential Algebraic Equations (IDAE). They are treated in the same manner as the ODE system of the previous section, following the idea of [12].

Hence, the second step is to recast the system in a quadratic format with the help of auxiliary variables $\mathbf{y}_{\mathrm{a}}$ :

$$
\mathbf{c}_{0}(t)+\lambda \mathbf{c}_{1}(t)+\lambda^{2} \mathbf{c}_{2}(t)+\mathbf{l}_{0}\left(\mathbf{y}_{\mathrm{f}}\right)+\lambda \mathbf{l}_{1}\left(\mathbf{y}_{\mathrm{f}}\right)+\mathbf{q}\left(\mathbf{y}_{\mathrm{f}}, \mathbf{y}_{\mathrm{f}}\right)+\mathbf{d}\left(\dot{\mathbf{y}}_{\mathrm{f}}\right)+\lambda \mathbf{d}_{1}\left(\dot{\mathbf{y}}_{\mathrm{f}}\right)+\mathbf{d} \mathbf{d}\left(\ddot{\mathbf{y}}_{\mathrm{f}}\right)=0
$$

The form of these equations generalizes the form (8). $\mathbf{c}_{0}, \mathbf{c}_{1}, \mathbf{c}_{2}, \mathbf{l}_{0}, \mathbf{l}_{1}, \mathbf{d}, \mathbf{d}_{1}, \mathbf{d d}, \mathbf{q}$ are respectively three constant operators, five linear operators and one bilinear operator. Again, this form as been chosen to take into account that $\lambda$ is constant in time.

Once this recast is available, the time-continuous system obtained (12) can be discretized automatically with different methods. The next two subsections 1.3.1 and 1.3.2 show how to obtain a quadratic algebraic system of the form (3) that can be continued with the ANM. Firstly, time domain discretization methods are discussed and secondly frequency domain methods. 


\subsubsection{Time-domain discretization methods}

Without any requirement on the type of solution sought for, an integration scheme can be applied to the quadratic system (12). Given a set of consistent initial conditions ${ }^{3} \mathbf{y}_{\mathrm{f}}(0), \dot{\mathbf{y}}_{\mathrm{f}}(0)$, the solution can be approximated on the grid $\{0, h, 2 h, \ldots, M h\}$ ( $h>0$ is the integration step and $M$ a given integer) for instance by a finite difference scheme with centered derivatives. For $0 \leq k \leq M-1$, the equations are then

$$
\begin{aligned}
& 0=\mathbf{c}_{0}(k h)+\lambda \mathbf{c}_{1}(k h)+\lambda^{2} c_{2}(k h)+\mathbf{l}_{0}\left(\mathbf{y}_{\mathrm{f}}(k h)\right)+\lambda \mathbf{l}_{1}\left(\mathbf{y}_{\mathrm{f}}(k h)\right)+\mathbf{q}\left(\mathbf{y}_{\mathrm{f}}(k h), \mathbf{y}_{\mathrm{f}}(k h)\right)+ \\
& \mathbf{d}\left(\frac{\mathbf{y}_{\mathrm{f}}((k+1) h)-\mathbf{y}_{\mathrm{f}}((k-1) h}{2 h}\right)+\lambda \mathbf{d}_{1}\left(\frac{\mathbf{y}_{\mathrm{f}}((k+1) h)-\mathbf{y}_{\mathrm{f}}((k-1) h}{2 h}\right)+\mathbf{d d}\left(\frac{\mathbf{y}_{\mathrm{f}}((k+1) h)-2 \mathbf{y}_{\mathrm{f}}(k h)+\mathbf{y}_{\mathrm{f}}((k-1) h}{h^{2}}\right)
\end{aligned}
$$

Like the system (12), this system is quadratic in the unknowns $\mathbf{y}_{\mathrm{f}}(m h), 0 \leq m \leq M$ and can be continued with the Asymptotic Numerical Method. In this case, the transcendental functions can be treated exactly in the same manner as briefly explained in section 3.4.

It is clear here that many other schemes could be used. The last example of this paper 3.4 describes an implementation of a Newmark scheme. In appendix B, time-integrations of a pendulum are performed with a class of Euler schemes that cover the explicit Euler, the implicit midpoint and the implicit Euler schemes. A collocation method could also be used to solve the system of equations, as done in [26] for the continuation of periodic solutions.

\subsubsection{Frequency-domain discretization methods}

Following the idea of [12] recalled in section 1.2, the method used to compute periodic solutions is here a purely frequency-domain method. The solutions of the quadratic system (12) are expanded in Fourier series (7) and after balancing the harmonics, in the same manner as for the equations (9), the system obtained is :

$$
\begin{aligned}
0= & \mathbf{C}_{0}+\lambda \mathbf{C}_{1}+\lambda^{2} \mathbf{C}_{2}+\mathbf{L}_{0}\left(\mathbf{Y}_{\mathrm{f}}\right)+\lambda \mathbf{L}_{1}\left(\mathbf{Y}_{\mathrm{f}}\right)+\mathbf{Q}\left(\mathbf{Y}_{\mathrm{f}}, \mathbf{Y}_{\mathrm{f}}\right)+ \\
& \omega \mathbf{D}\left(\mathbf{Y}_{\mathrm{f}}\right)+\lambda \omega \mathbf{D}_{1}\left(\mathbf{Y}_{\mathrm{f}}\right)+\omega^{2} \mathbf{D D}\left(\mathbf{Y}_{\mathrm{f}}\right)
\end{aligned}
$$

The two last terms of this system are cubic in the unknowns $\left(\mathbf{Y}_{\mathrm{f}}, \omega, \lambda\right)$ of the system. Hence, two additional auxiliary variables are introduced $\Lambda=\lambda \omega$ and $\Omega=\omega^{2}$ to obtain the final quadratic recast

$$
\begin{aligned}
0= & \mathbf{C}_{0}+\lambda \mathbf{C}_{1}+\lambda^{2} \mathbf{C}_{2}+\mathbf{L}_{0}\left(\mathbf{Y}_{\mathrm{f}}\right)+\lambda \mathbf{L}_{1}\left(\mathbf{Y}_{\mathrm{f}}\right)+\mathbf{Q}\left(\mathbf{Y}_{\mathrm{f}}, \mathbf{Y}_{\mathrm{f}}\right)+ \\
& \omega \mathbf{D}\left(\mathbf{Y}_{\mathrm{f}}\right)+\Lambda \mathbf{D}_{1}\left(\mathbf{Y}_{\mathrm{f}}\right)+\Omega \mathbf{D D}\left(\mathbf{Y}_{\mathrm{f}}\right) \\
0= & \Omega-\omega^{2} \\
0= & \Lambda-\lambda \omega
\end{aligned}
$$

As opposed to the time-domain discretization method, the transcendental nonlinearities cannot be treated directly with the formalism recalled in section 1.1.2 that is detailed in the book [21] and the article [22]. The reference [21] states clearly that it is sufficient to treat the case of an auxiliary variable $v(t)=\mathrm{g}(u(t))$ where $\mathrm{g}$ is a transcendental function, which is defined with a differential system of the form :

$$
\begin{aligned}
v(0) & =\mathrm{g}(u(0)) \\
\dot{v}(t) & =w(t) \dot{u}(t)
\end{aligned}
$$

where the variables $u, v$ and $w$ are periodic functions and $\dot{\mathrm{g}}(u(t))=w(t)$. The final quadratic recast with $\mathbf{U}, \mathbf{V}, \mathbf{W}$ being the vectors of Fourier coefficients of $u, v, w$ respectively is

$$
\begin{aligned}
v(0) & =\mathrm{g}(u(0)) \\
\mathrm{d} \mathbf{L}(\mathbf{V}) & =\mathrm{d} \mathbf{Q}(\mathbf{W}, \mathbf{U})
\end{aligned} \quad \text { and } \quad \mathrm{d} v(0)=w(0) \mathrm{d} u(0)
$$

where $\mathrm{d} \mathbf{L}$ and $\mathrm{d} \mathbf{Q}$ are suitable linear and bilinear operators respectively. The details on this algebra are given in appendix $\mathrm{A}$.

The generalization of this approach to quasi-periodic solutions is explained in [24]. The solutions are expressed as double truncated Fourier series of the form

$$
\mathbf{y}(t)=\mathbf{Y}_{0}+\sum_{k_{1}=1}^{H_{1}} \sum_{k_{2}=1}^{H_{2}}\left(\mathbf{Y}_{c, k_{1}, k_{2}} \cos \left(k_{1} \omega_{1} t+k_{2} \omega_{2} t\right)+\mathbf{Y}_{s, k_{1}, k_{2}} \sin \left(k_{1} \omega_{1} t+k_{2} \omega_{2} t\right)\right) .
$$

As there are two independent angular frequencies, an additional phase condition must be added to solve the algebraic system obtained after balancing the harmonics. For brevity the algebra is not detailed in this paper. The interested reader is referred to the article [24] or to the PhD thesis [41] for further details.

\footnotetext{
3 In the case of a system with delay or fractional order derivatives, it may be required to give the initial conditions over an interval of the form $[-T, 0]$.
} 


\section{Periodic solutions of ODE, DAE, IDE, DDE and fractional order systems}

In this section, periodic solutions of ODE, DAE, IDE, DDE and fractional order systems are continued. Conservative and non-conservative systems are both treated. The functions appearing below are supposed analytic on their domain of definition. As the solutions investigated in this section are all periodic, all the functions of the time appearing are supposed periodic. The example of the pendulum is treated in three different formulations : solving the polar coordinates equations of motion, solving the Cartesian coordinates equations of motion and solving the equation of conservation of the energy. This illustrates the ability to cope with ODE, DAE and IDE in the same framework and to check that the results are consistent. A Van der Pol oscillator is then treated with different feedbacks, to show the application of the methods to fractional order systems and to delay differential equations.

\subsection{Ordinary Differential Equations : Usual polar coordinates pendulum}

The ODE are a very widespread type of equations that can be given in the following form:

$$
\dot{\mathbf{y}}=\mathbf{f}(\mathbf{y}, t), \quad \text { where } \mathbf{y} \text { and } \mathbf{f}(\mathbf{y}, t) \in \mathbb{R}^{n}
$$

The equations of the motions of the pendulum in polar coordinates are given by:

$$
\left\{\begin{array}{l}
\dot{\theta}=\phi \\
\dot{\phi}=-\sin (\theta)-\lambda \phi
\end{array}\right.
$$

where $\lambda \phi$ is an unfolding term [35] added to compute the family of periodic solutions easily. Once the system solved, $\lambda$ is found to be zero since no periodic solution exists otherwise. These equations are recast quadratically, in the form of equations (12) and (16) :

$$
\left\{\begin{aligned}
\dot{\theta} & =\phi \\
\dot{\phi} & =-s-\lambda \phi \\
s(0) & =\sin (\theta(0)) \text { and } \dot{s}=c \dot{\theta} \\
c(0) & =\cos (\theta(0)) \text { and } \dot{c}=-s \dot{\theta}
\end{aligned}\right.
$$

These equations are treated automatically with the method proposed in section 1.3.2. The results are shown and discussed in section 2.4.

\subsection{Differential Algebraic Equations : Cartesian coordinates pendulum}

The DAE are a type of equations where there are both differential equations and algebraic equations between the unknowns. It can be given in the following form:

$$
\left\{\begin{aligned}
\dot{\mathbf{y}}_{1} & =\mathbf{f}\left(\mathbf{y}_{1}, \mathbf{y}_{2}, t\right), \quad \text { where } \mathbf{y}_{1}, \mathbf{f}\left(\mathbf{y}_{1}, \mathbf{y}_{2}, t\right) \in \mathbb{R}^{n_{\text {diff }}} \text { and } \mathbf{y}_{2}, \mathbf{g}\left(\mathbf{y}_{1}, \mathbf{y}_{2}, t\right) \in \mathbb{R}^{n_{\text {alg }}} \\
0 & =\mathbf{g}\left(\mathbf{y}_{1}, \mathbf{y}_{2}, t\right)
\end{aligned}\right.
$$

The equations of the motions of the pendulum in Cartesian coordinates are given by:

$$
\left\{\begin{aligned}
\ddot{x}+\lambda \dot{x}+T x & =0 \\
\ddot{y}+\lambda \dot{y}+T y-1 & =0 \\
x^{2}+y^{2}-1 & =0
\end{aligned}\right.
$$

where $T$ is the tension of the string, $x, y$ are respectively the horizontal and the vertical position of the pendulum. Similarly to the previous case, $\lambda \dot{x}$ and $\lambda \dot{y}$ are unfolding terms [35] added to compute the family of periodic solutions easily. Once the system solved, $\lambda$ is found to be zero since no periodic solution exists otherwise. These equations are already quadratic according to equations (12) and are then treated automatically with the method proposed in section 1.3.2. The results are shown and discussed in section 2.4. 


\subsection{Implicit Differential Equations : Conservation of the Energy}

The IDE are a type of equations where the derivatives cannot be expressed explicitly (as for the ODE (19)). It can be written

$$
\mathbf{f}(\dot{\mathbf{y}}, \mathbf{y}, t)=0, \quad \text { where } \mathbf{y}, \mathbf{f}(\dot{\mathbf{y}}, \mathbf{y}, t) \in \mathbb{R}^{n}
$$

The potential energy of the pendulum is defined as $-1-\cos (\theta)$ so that its maximum value for periodic orbits is 0 . The equation of conservation of the total energy of the pendulum is given by:

$$
\frac{\dot{\theta}^{2}}{2}-1-\cos (\theta)=\lambda
$$

where $\lambda$ is the energy of the pendulum. Its quadratic recast is given by:

$$
\left\{\begin{aligned}
\frac{\phi^{2}}{2}-1-c & =\lambda \\
\phi & =\dot{\theta} \\
s(0) & =\sin (\theta(0)) \text { and } \dot{s}=c \dot{\theta} \\
c(0) & =\cos (\theta(0)) \text { and } \dot{c}=-s \dot{\theta}
\end{aligned}\right.
$$

These equations are compatible with equations (12) and (16) and are then treated automatically with the method proposed in section 1.3.2. The results are shown and discussed in section 2.4.

\subsection{Bifurcation diagram of the pendulum}

The branch of periodic solutions of the pendulum is represented on the bifurcation diagrams in figure 1 . The relative error $\epsilon$ between the theoretical value $T_{\text {th }}$ and the computed value $T$ of the period of the oscillations is given by $\epsilon=\left|\frac{T_{\mathrm{th}}-T}{T_{\mathrm{th}}}\right|$. The theoretical value is given by $T_{\mathrm{th}}=4 K\left(\sin \left(\frac{\max (\theta)}{2}\right)^{2}\right)$ where $K$ is the complete elliptic integral of the first kind (see [33] or [30] for what could be the first derivations of this formula). The relative error gets bigger while the energy gets closer to its maximum value 0 . It is probably because the computation would need more than just a double precision setting and the solution more than $H=500$ harmonics to be well represented in this area. The periodic solution represented in figure 2 shows a solution with an energy equal to $-10^{-14}$, very close to its maximum value 0 . We recall here that the reference of the potential energy of the system has been taken so that the maximal value of the total energy of a periodic solution is 0 .
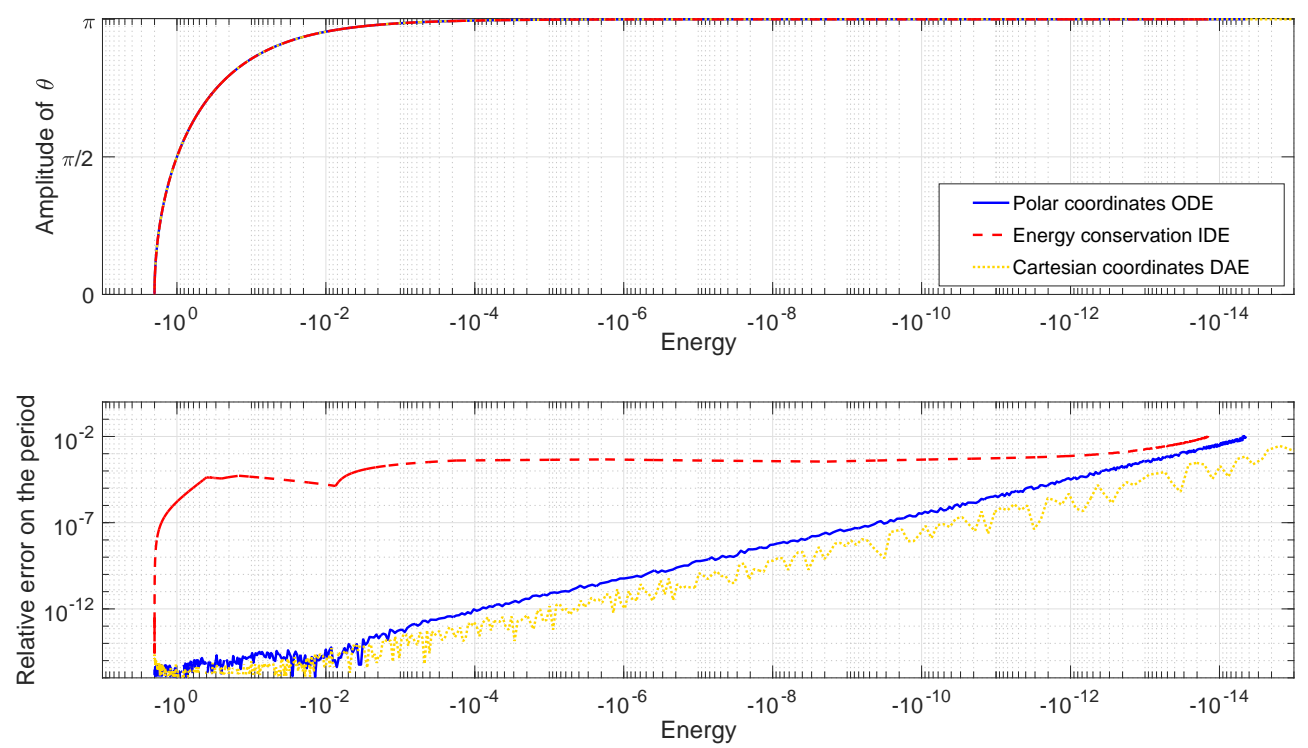

Fig. 1 A bifurcation diagram of the pendulum with $H=500$. The top figure represents the amplitude of the oscillations with respect to the energy. The bottom figure shows the relative error between the theoretical value (see text) and the computed value of the period of the oscillations in logarithmic scales. In blue the ODE system (21) is solved, in dashed red the DAE system (23) and in dotted yellow the IDE system (26). 


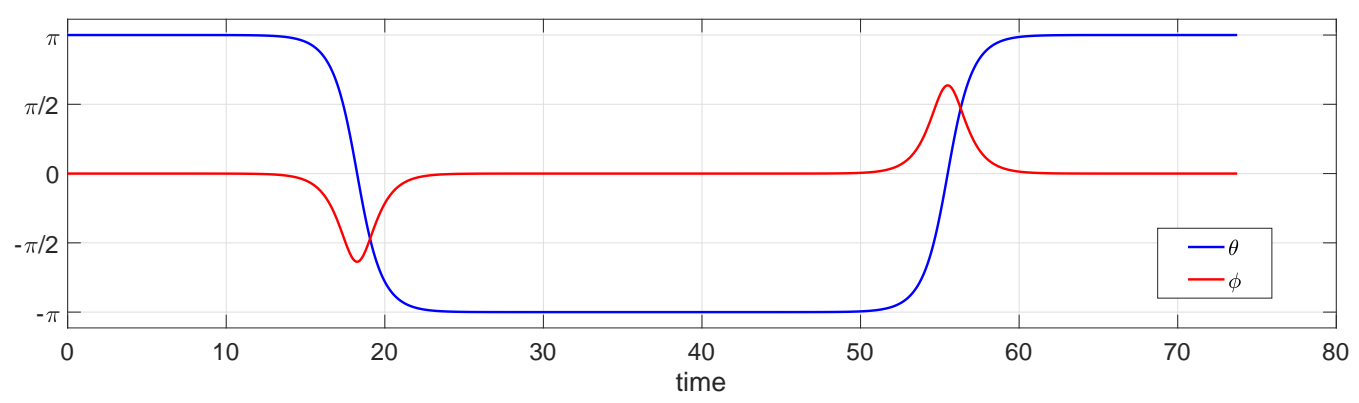

Fig. 2 A solution of the pendulum with the energy equals to $-10^{-14}$ (that is very close to its maximum value 0 ) and $H=500$ harmonics. It is almost a homoclinic orbit in the sense that it remains a very long time very close to the unstable equilibrium $\theta=\pi($ or $-\pi)$.

The three bifurcation diagrams for the three different formulations are superimposed on the top figure 1 . It is not possible to distinguish the three bifurcation diagrams. On the bottom figure 1 the relative errors between the analytic reference for the period and the one computed by our method are shown. This error remains very small (under one percent) but shows that the three formulations do not give exactly the same results. This is probably due on the one hand to the numerical accuracy and on the other hand to the system solved that are not exactly the same.

The time computation for ten continuation steps is around 45 seconds for each system. The bifurcation diagrams obtained with ODE and DAE formulations require 50 continuation steps and the one with IDE formulation 75 continuation steps. The IDE formulation (25) has directly the energy as its continuation parameter. The DAE formulation (23) gives the tension of the string without post-processing as it is an unknown of the equations. The ODE formulation (20) can be used to compute the stability of the solutions obtained. Most of the time, this is the only way to have access to the stability informations of a system as a lot more results are known for ODE than for other types of systems. It seems that the DAE formulation is more robust when the solution gets closer to the homoclinic orbit, that is the limit where the period of the oscillations tends to infinity (and the energy, as defined in (25), to zero). This can be seen through the relative error on the period of the oscillations on the top of figure 1 that is everywhere smaller than for the other systems solved. The reason behind this is not clear yet for the authors.

\subsection{Delay Differential Equations and fractional order systems}

Two other types of systems have been studied using ANM and HBM. The delay differential systems [23] and the systems with fractional order derivatives [42]. To summarize these type of systems, a Van der Pol oscillator with feedback is explored

$$
\ddot{x}-\varepsilon\left(1-x^{2}\right) \dot{x}+x=-k \varepsilon F(x)
$$

where $F(x)=x_{\tau}$ or $D^{\alpha}(x)$ with $D^{\alpha}$ the operator of Weyl fractional order derivation of order $\alpha$ and $x_{\tau}(t)=$ $x(t-\tau)$. To recast these equations quadratically, three auxiliary variables $y=\dot{x}, r=1-x^{2}$ and $F_{\mathrm{n} l}=r y-k F(x)$ are introduced. This gives :

$$
\begin{array}{ll}
\dot{y}+x+\varepsilon F_{\mathrm{n} l} & =0 \\
y-\dot{x} & =0 \\
r-1+x^{2} & =0 \\
F_{\mathrm{n} l}-k F(x)+r y & =0
\end{array}
$$

Since equations (28) is quadratic according to the framework defined by equations (12), the results are obtained automatically according to the process detailed in section 1.3.2. The figure 3 shows two bifurcation diagrams computed with the system (28), where the amplitude of the solution $x$ of (27) is represented with respect to the continuation parameter which is $\tau$ or $\alpha$ depending on whether $F(x)=x_{\tau}$ or $F(x)=D^{\alpha}(x)$ respectively. The two bifurcation diagrams are completely different. There are two Hopf bifurcations ${ }^{4}$ for the fractional order system and it seems that there are two vertical asymptotes at $\alpha=-1$ and $\alpha=3$. It is

${ }^{4}$ Following [42], we use here the name Hopf bifurcation. To our knowledge, this type of bifurcation has not been characterized theoretically but is analogous to a Hopf bifurcation point from a numerical point of view. 
remarkable that the method still gives the results for $\alpha$ negative. The value $\alpha=-1$ allows to solve integrodifferential equations with our method. The delay differential system shows only one Hopf bifurcation in the area represented in figure 3. Even when $\tau$ is negative, the method gives some results which would not be possible to obtain with standard solvers as this represents a feedback from the future : the system is not causal anymore.

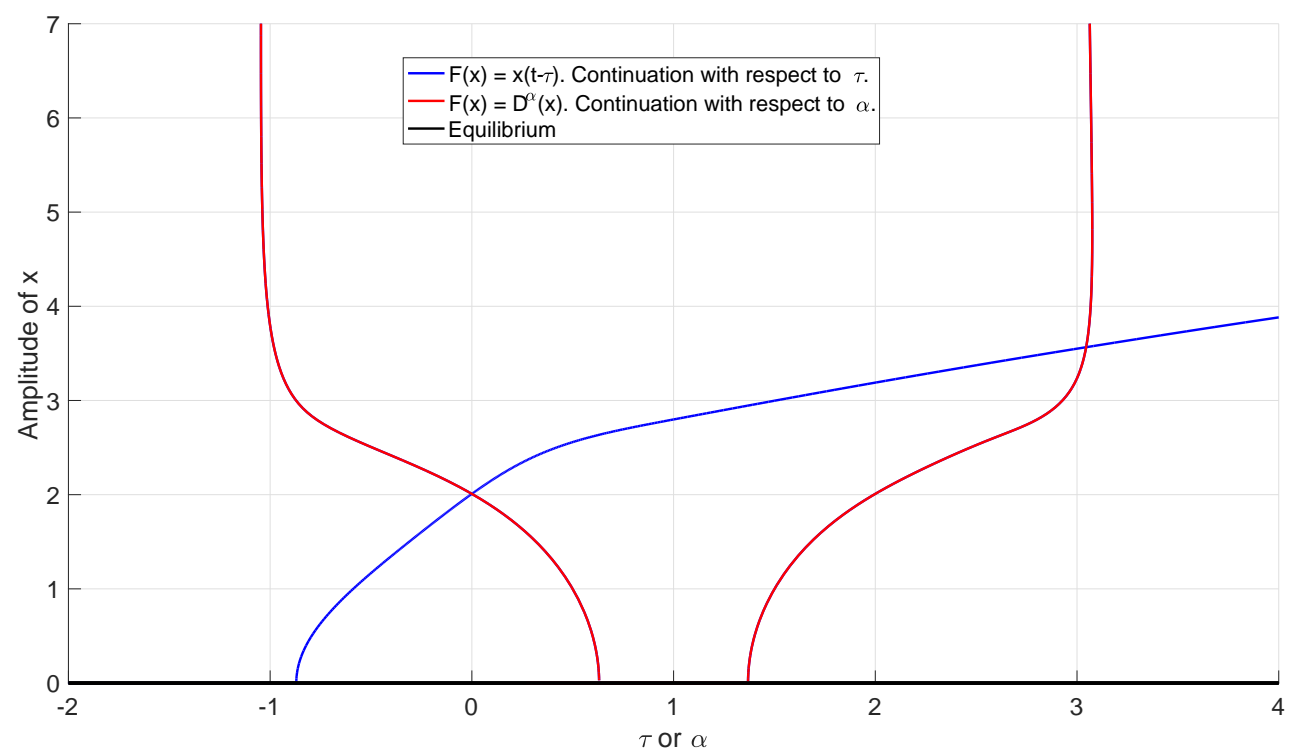

Fig. 3 The solution branches of (27) with $\varepsilon=2$ and $k=1.5$ and for $F(x)=x(t-\tau)$ in blue and $F(x)=D^{\alpha}(x)$ in red. The continuation parameter is respectively $\tau$ and $\alpha$. The blue point and the red point with abscissa 0 coincide since both systems are equal : $x(t-0)=x(t)=D^{0}(x)(t)$.

\section{Equilibrium, periodic, quasi-periodic and transients regimes of an ODE}

This section shows the different types of solutions that can be obtained with our method. For the sake of clarity, an academic example consisting in a system of coupled Van der Pol oscillators is studied in detail. This example has been used to illustrate the quasi-periodic Harmonic Balance Method (QPHBM) in [24]. The equations are

$$
\begin{aligned}
& \dot{x}_{1}+a_{1} \dot{x}_{1}+\Omega_{1}^{2} x_{1}=\left(b_{1} \lambda-c_{1} \lambda\left(x_{1}+x_{2}\right)-d_{1} \lambda\left(x_{1}+x_{2}\right)^{2}\right)\left(\dot{x}_{1}+\dot{x}_{2}\right) \\
& \dot{x}_{2}+a_{2} \dot{x}_{2}+\Omega_{2}^{2} x_{2}=\left(b_{2} \lambda-c_{2} \lambda\left(x_{1}+x_{2}\right)-d_{2} \lambda\left(x_{1}+x_{2}\right)^{2}\right)\left(\dot{x}_{1}+\dot{x}_{2}\right)
\end{aligned}
$$

The value of the dimensionless parameters are $\Omega_{1}=1, a_{1}=0.01, b_{1}=0.5, c_{1}=d_{1}=2 ; \Omega_{2}=2.5, a_{2}=0.025$, $b_{2}=1, c_{2}=d_{2}=4$. The first order quadratic recast is the one derived in [24] :

$$
\begin{aligned}
\dot{x}_{1} & =y_{1} \\
\dot{y}_{1} & =-a_{1} y_{1}-\Omega_{1}^{2} x_{1}+b_{1} \lambda\left(y_{1}+y_{2}\right)-c_{1} \lambda w-d_{1} \lambda v \\
\dot{x}_{2} & =y_{2} \\
\dot{y}_{2} & =-a_{2} y_{2}-\Omega_{2}^{2} x_{2}+b_{2} \lambda\left(y_{1}+y_{2}\right)-c_{2} \lambda w-d_{2} \lambda v \\
0 & =r-\left(x_{1}+x_{2}\right)^{2} \\
0 & =w-\left(x_{1}+x_{2}\right)\left(y_{1}+y_{2}\right) \\
0 & =v-\left(x_{1}+x_{2}\right) \times w
\end{aligned}
$$

Now these quadratic equations (in the format (12) previously defined) will be used to continue equilibrium, periodic solutions, quasi-periodic solutions and transient regimes of the system. For equilibrium or periodic solutions, the stable solutions are represented in solid lines and the unstable solutions in dotted lines. 


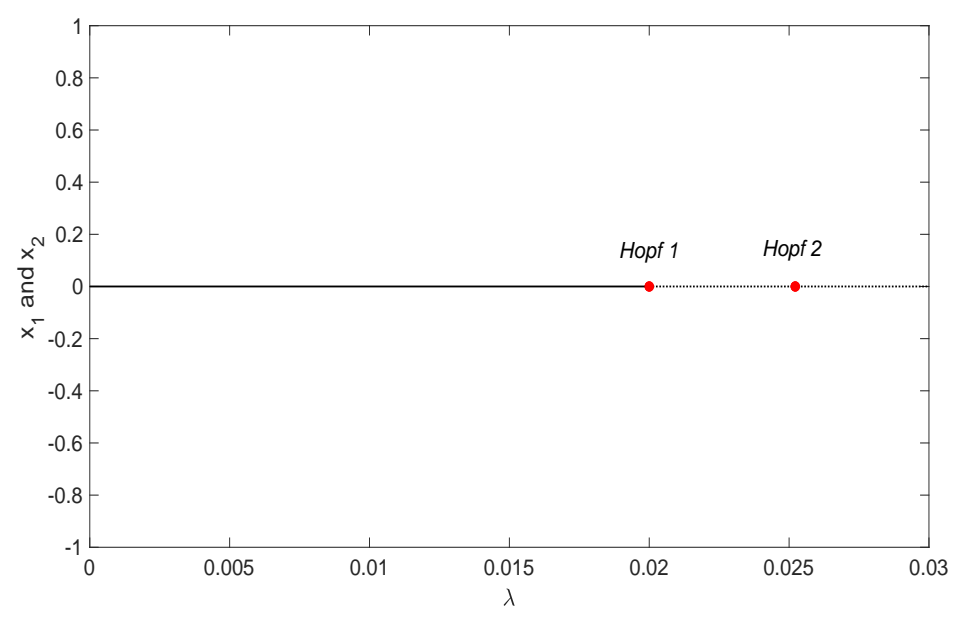

Fig. 4 A bifurcation diagram of the equilibrium of system (30). The Hopf bifurcations are represented by red dots.

\subsection{Equilibrium}

In the quadratic recast (31), the derivatives are replaced by 0 to obtain the system to solve to find the equilibrium. This system is obtained (easily) automatically from (31). It is clear here that the equilibrium is just $x_{1}=y_{1}=x_{2}=y_{2}=r=w=v=0$. The stability of this equilibrium requires the computation of the eigenvalues of the Jacobian matrix of the first order system. This computation is automatized with the help of the condensation operation, detailed in [22]), and gives two Hopf bifurcations for $\lambda=0.02$ and $\lambda \simeq 0.025$ as shown in figure 4.

\subsection{Periodic solutions}

The quadratic recast (31) is used for periodic solutions as explained in section 1.3.2. From the Hopf bifurcations computed in the previous section, two periodic orbits arise that can be continued. The starting point is computed automatically using the eigenvectors and the eigenvalues of the Jacobian matrix at the bifurcation point [3]. The stability of the periodic orbit is given by the Floquet exponents of the systems, computed with Hill's method as explained in [31] and [2]. With this system two Neimark-Sacker bifurcations are detected, one on each branch, for $\lambda \simeq 0.03$ on the branch arising from the second Hopf bifurcation and for $\lambda \simeq 0.35$ on the branch arising from the first Hopf bifurcation. The bifurcation diagram obtained is represented on figure 5 .

\subsection{Quasi-periodic solutions}

The quadratic recast (31) is also used here for quasi-periodic solutions. They are represented by double Fourier series (18), using the Quasi-Periodic Harmonic Balance Method (QPHBM) to write the equations (31) in the frequency domain. The QPHBM has been explained in [24] using this same example. In this same reference, the reader will find the explanation on how to obtain an algebraic system automatically to continue the quasi-periodic solutions. The bifurcation diagram obtained is represented on figure 6 .

Only one branch of quasi-periodic solutions that connects the two bifurcations (see figure 6) can be computed from the Neimark-Sacker bifurcations. The starting point is computed automatically from the eigenvectors of Hill's matrix and the Floquet exponents at the bifurcation point (not detailed here). The question of the stability of a quasi-periodic orbit is a tough problem that is not investigated here.

\subsection{Transient regimes}

In this section, the system of equations (31) is discretized on time samples : $0, h, 2 h, \ldots, T-h, T$ where $T=400$ and the integration step is $h=\frac{T}{N-1}, N \in \mathbb{N}$. With this definition the number of time samples is $N$ which is set to 2000 in the applications. The algebraic system is obtained by writing a Newmark scheme [37] in the way explained in section 1.3.1, that is, for all $n \geq 1$ : 


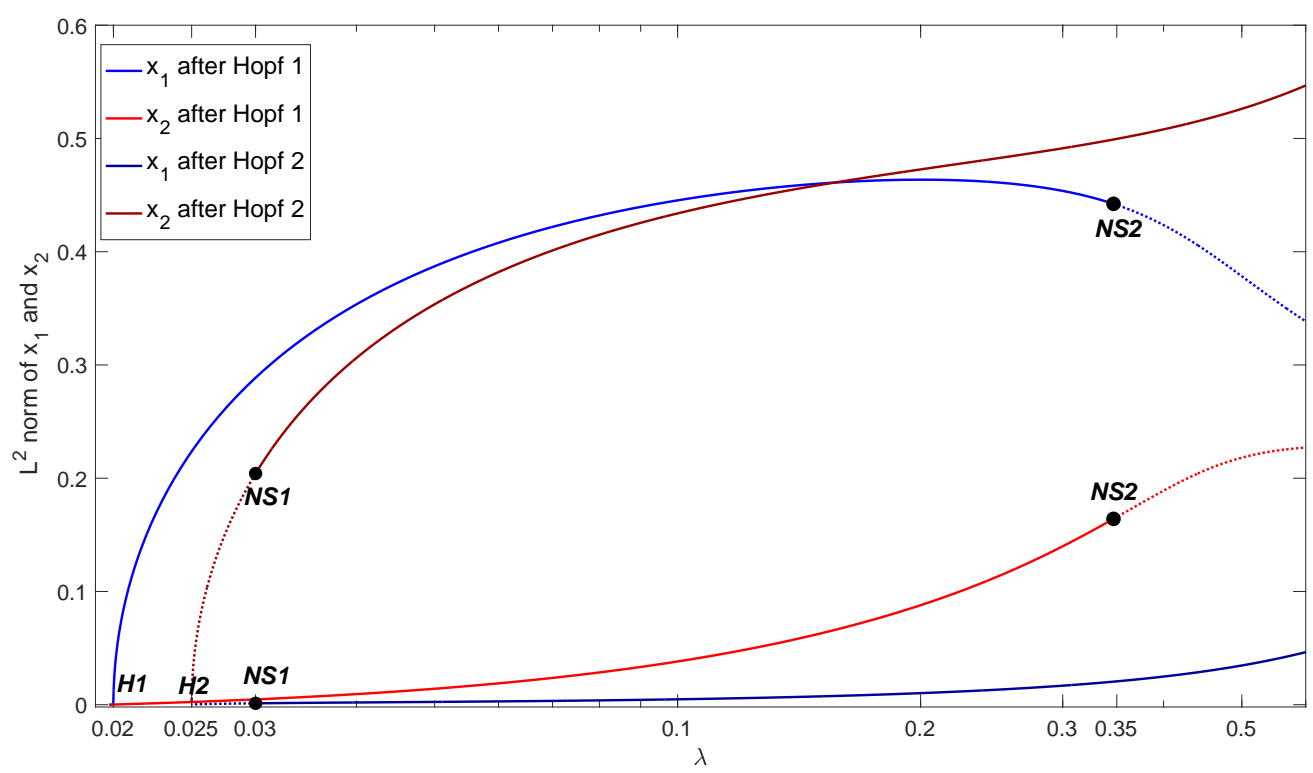

Fig. 5 A bifurcation diagram of the periodic solutions of the system (30). The x-axis is in logscale to help to distinguish the two branches arising from $\mathrm{H} 1$ and $\mathrm{H} 2$, which are the first and the second Hopf bifurcations shown on figure 4 . NS1 and NS2 are the two Neimark-Sacker bifurcations detected.

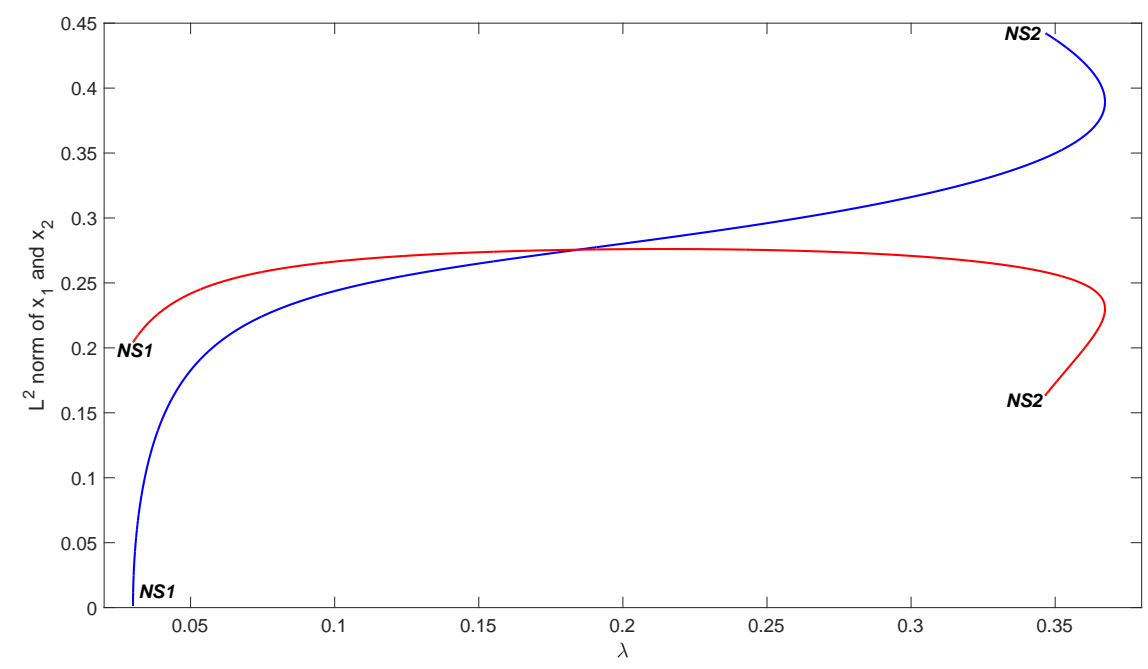

Fig. 6 A bifurcation diagram showing the quasi-periodic branch of the system (30). NS1 and NS2 are the two Neimark-Sacker bifurcations shown on figure 5 .

$$
\begin{aligned}
x_{1}(n h) & =x_{1}((n-1) h)+h y_{1}((n-1) h)+h^{2}\left(\left(\left(\frac{1}{2}-\beta\right) \ddot{x}_{1}((n-1) h)+\beta \ddot{x}_{1}(n h)\right)\right. \\
y_{1}(n h) & =y_{1}((n-1) h)+h(1-\gamma) \ddot{x}_{1}((n-1) h)+h \gamma \ddot{x}_{1}(n h) \\
x_{2}(n h) & =x_{2}((n-1) h)+h y_{2}((n-1) h)+h^{2}\left(\left(\left(\frac{1}{2}-\beta\right) \ddot{x}_{2}((n-1) h)+\beta \ddot{x}_{2}(n h)\right)\right. \\
y_{2}(n h) & =y_{2}((n-1) h)+h(1-\gamma) \ddot{x}_{2}((n-1) h)+h \gamma \ddot{x}_{2}(n h) \\
r(n h) & =\left(x_{1}(n h)+x_{2}(n h)\right)^{2} \\
w(n h) & =\left(x_{1}(n h)+x_{2}(n h)\right)\left(y_{1}(n h)+y_{2}(n h)\right) \\
v(n h) & =\left(x_{1}(n h)+x_{2}(n h)\right) \times w(n h)
\end{aligned}
$$

$\beta$ and $\gamma$ are the parameters of the scheme and will be set to $\frac{1}{4}$ and $\frac{1}{2}$ respectively for the computations. The second order derivatives are given by :

$$
\begin{aligned}
& \ddot{x}_{1}(n h)=-w_{1}^{2} x_{1}(n h)-a_{1} y_{1}(n h)+\left(b_{1} \lambda\right)\left(y_{1}(n h)+y_{2}(n h)\right)-\left(c_{1} \lambda\right) w(n h)-\left(d_{1} \lambda\right) v(n h) \\
& \ddot{x}_{2}(n h)=-w_{2}^{2} x_{2}(n h)-a_{2} y_{2}(n h)+\left(b_{2} \lambda\right)\left(y_{1}(n h)+y_{2}(n h)\right)-\left(c_{2} \lambda\right) w(n h)-\left(d_{2} \lambda\right) v(n h)
\end{aligned}
$$


Initial conditions are added to the system (32). They are set to $x_{1}(0)=x_{2}(0)=y_{2}(0)=0$ and $y_{1}(0)=0.01$. It should be noticed that the system (32) can be automatically obtained thanks to the quadratic recast (31). The transient solutions are continued with respect to $\lambda$. Instead of a bifurcation diagram, the figure 7 represents all the transient solutions along the solution branch in a waterfall plot.
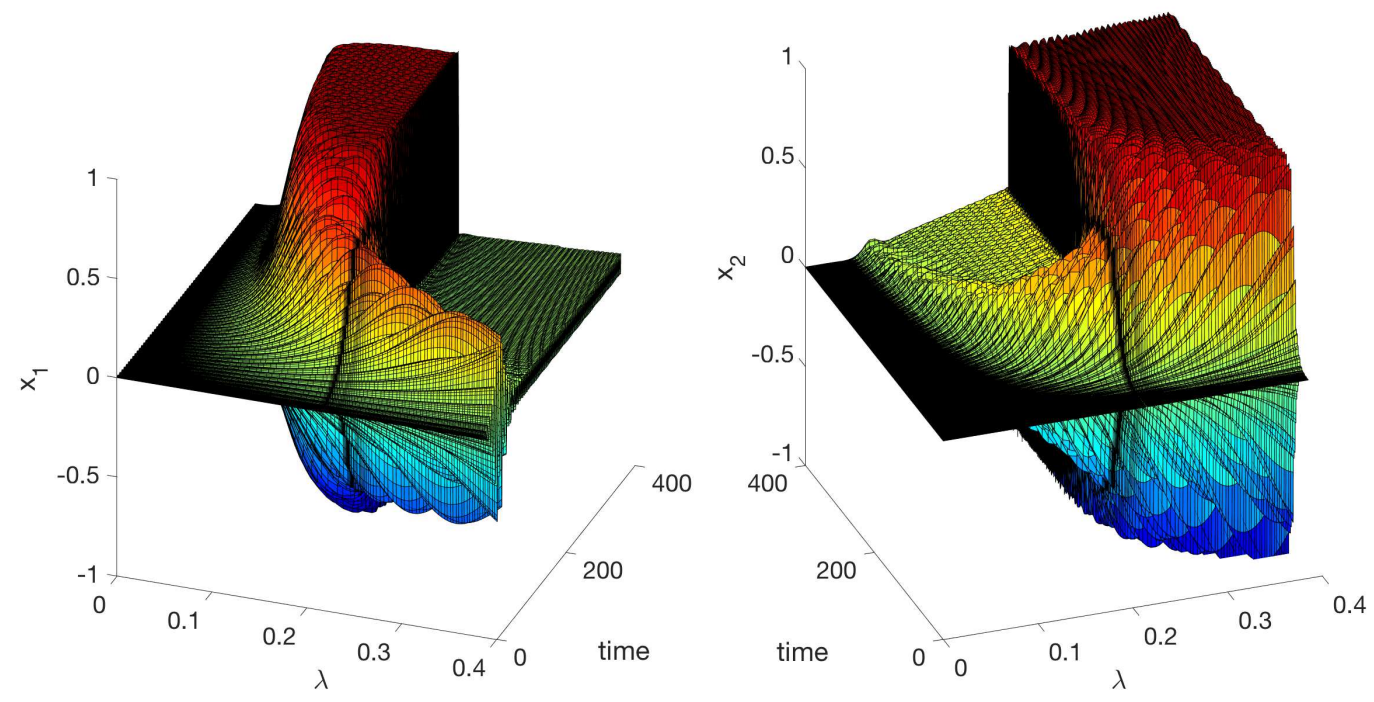

Fig. 7 Two waterfalls of the transients of (30). The left figure represents the motion of the first oscillator (of resonance frequency $\Omega_{1}$ ) and the right figure the motion of the second oscillator (of resonance frequency $\Omega_{2}$ ). For small values of $\lambda$, the transients vanish towards the stable Equilibrium. For larger values of $\lambda$, the transients first converge towards a periodic orbit at pulsation around $\Omega_{1}$, then there is a narrow transition zone where the transients become very long and finally, for large values of $\lambda$ the transients converge towards a periodic orbit at a pulsation around $\Omega_{2}$.

For small values of $\lambda \lesssim 0.05$, the oscillations are either evanescent $\lambda<0.02$ or very slowly growing to a limit cycle that would need a longer time of integration to be reached. Between $\lambda \simeq 0.05$ and $\lambda \simeq 0.2144$, the solutions tend to the first limit cycle, which has a frequency similar to the resonance frequency of the first oscillator, $\Omega_{1}$. For $\lambda \gtrsim 0.2144$, the solutions tend to the second limit cycle, which has a frequency similar to resonance frequency of the second oscillator, $\Omega_{2}$. When $\lambda \simeq 0.2144$, the transients become very long and look very much like the quasi-periodic regimes found in the previous section. However, as this is only a timeintegration algorithm, one should not forget that these results depend on the time of integration and on the integration step. When the number of time samples is increased to $N=4000,6000,8000$, this quasi-periodic transition occurs for $\lambda \simeq 0.1780,0.1720,0.1700$ respectively.

\subsection{Phase space representation}

The different types of solutions continued in the previous sections are represented altogether in 2-dimensional and 3-dimensional projections of the 4 -dimensional phase space $\left(x_{1}, y_{1}, x_{2}, y_{2}\right)$ in figure 8 . Two transient trajectories are represented together with the two stable limit cycles and the quasi-periodic orbit. The two transients were computed with slightly different initial conditions : for both, $x_{2}(0)=y_{1}(0)=y_{2}(0)=0$. The transient that converges towards the limit cycle at $\Omega_{1}$ (in blue, in the center of the 3 -D projection) has been computed with $x_{1}(0)=10^{-3}$ and the other one, in red, that converges towards the limit cycle at $\Omega_{2}$, with $x_{1}(0)=10^{-2}$. As the 3-D projection is in the space $\left(x_{2}, y_{2}, y_{1}\right)$, the two transients start from the same point, but this is of course not the case in the full 4-D phase space.

All the solutions represented in figure 8 have been computed using a unique code developed by the authors. The value of the continuation parameter is $\lambda=0.2144$. 

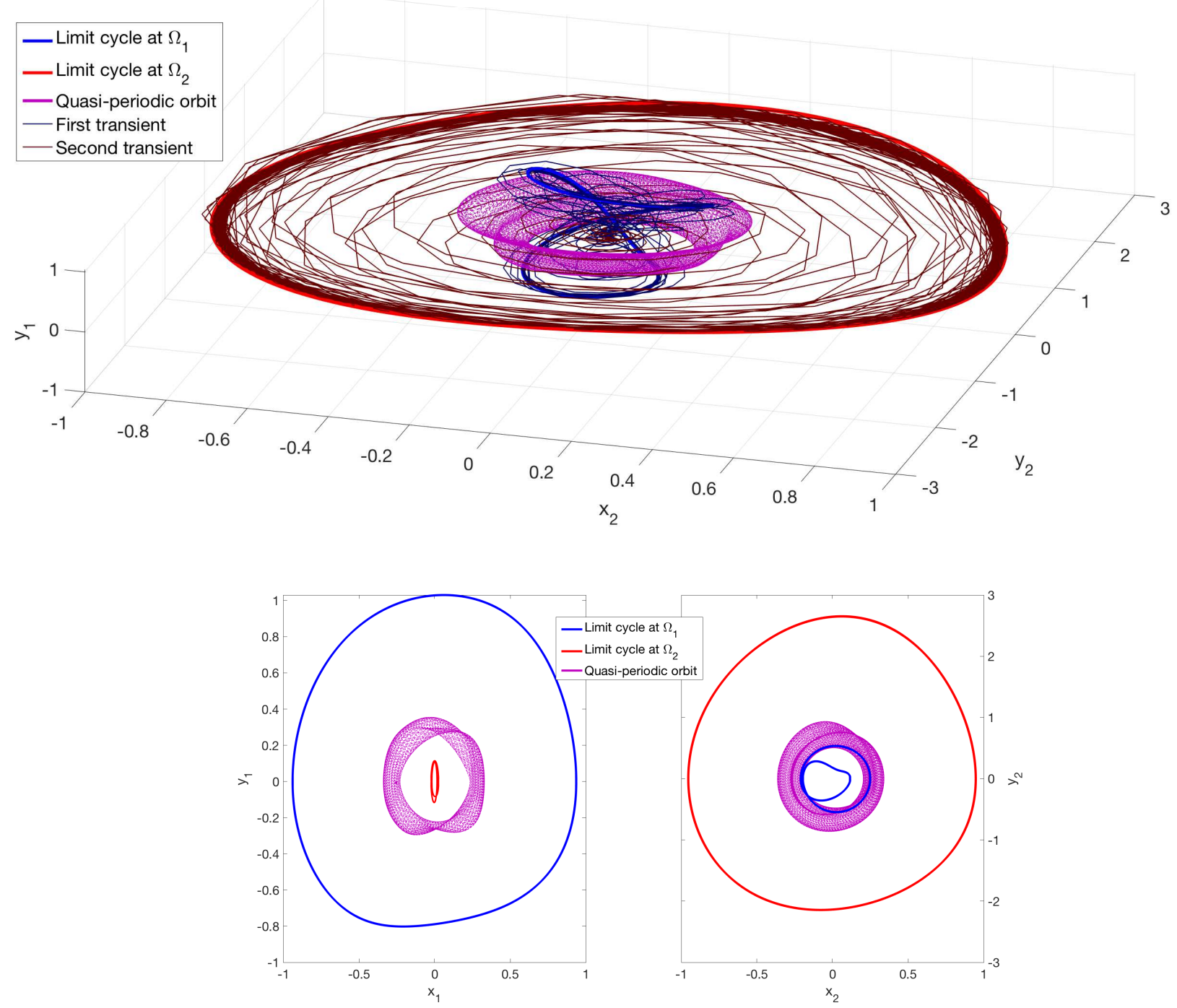

Fig. 8 These figures show three different projections of the phase space for $\lambda=0.2144$. The quasi-periodic orbit is in pink while the two limit cycles associated with the pulsation $\Omega_{1}$ and $\Omega_{2}$ are in blue and in red respectively. The transients in dark blue and dark red converge respectively to the blue and red limite cycles.

\section{Conclusion and perspectives}

This article shows how the quadratic recast of any smooth nonlinear system can be applied to continue its equilibrium, periodic or quasi-periodic solutions as well as its transients with the Asymptotic Numerical Method (ANM). The approach presented has been shown to be applicable to ordinary differential equations, delay differential equations, implicit differential algebraic equations and fractional order equations. The solutions of these systems are continued using the same implementation of the ANM. Therefore, further developments of the ANM benefit directly to all the continuation problems handled, requiring very few modifications. The academic examples proposed have illustrated the genericity of the framework described. An object-oriented implementation of the method has been developed ${ }^{5}$ under the name Manlab. The code Manlab tries to unify the treatment of the large variety of solutions and systems presented in this paper. From a user point of view as well as from a developer point of view, there is a minimum of work to do to switch from a type of system to another and from a type of solution to another. The crucial point is to provide a quadratic recast of the dynamical systems, most of the other operations have been automatized or could be automatized.

The perspectives of this work are many. The global continuation of an integration scheme offers the possibility to study its properties from a numerical point of view as can be seen in appendix B. The attractors of a system can be sought for by continuation with respect to the initial conditions. Chaotic areas could be investigated using the sensibility of the continuation to the initial conditions. The high robustness of the con-

\footnotetext{
5 Manlab is available online on the dedicated website https://manlab.lma.cnrs-mrs.fr/ .
} 
tinuation with the ANM is clearly an advantage to treat in a unified framework a wide range of problems coming from either purely algebraic equations or time domain and frequency domain discretizations of continuous equations. The comparison of several standard methods of representation of the solutions of a system is possible, allowing global comparison between direct time integration, harmonic balance, orthogonal polynomial collocation, trigonometric collocation, alternating frequency time scheme, etc... in the same framework.

Acknowledgements This work has been carried out in the framework of the Labex MEC (ANR-10-LABX-0092) and of the A*MIDEX project (ANR-11-IDEX-0001-02), funded by the Investissements d'Avenir French Government program managed by the French National Research Agency (ANR). Conflict of Interest: The authors declare that they have no conflict of interest.

\section{A Algebra of the HBM applied on transcendental functions}

The case of an auxiliary variable $v$ defined as $v(t)=\mathrm{g}(u(t))$ is treated in detail. Let us suppose that the auxiliary variable $w(t)=\dot{\mathrm{g}}(u(t))$ has been rewritten quadratically ${ }^{6}$, the variable $v$ can be defined as the unique solution of the differential system

$$
\begin{aligned}
& v(0)=\mathrm{g}(u(0)) \\
& \dot{v}(t)=w(t) \dot{u}(t)
\end{aligned}
$$

which is the same as (16). The auxiliary variables $u_{0}=u(0), v_{0}=v(0)$ and $w_{0}=w(0)$ are introduced to recast the first equation quadratically with the system :

$$
\begin{aligned}
v_{0} & =\mathrm{g}\left(u_{0}\right) \\
\mathrm{d} v_{0} & =w_{0} \mathrm{~d} u_{0}
\end{aligned} .
$$

This system is treated with the formalism explained in [22] briefly recalled in section 1.1.2. For the second equation, let us define the complex truncated Fourier series of $u, v$ and $w$ by :

$$
u(t)=\sum_{h=-H}^{H} \mathbf{U}_{h} \exp (i h \omega t), v(t)=\sum_{h=-H}^{H} \mathbf{V}_{h} \exp (i h \omega t) \text { and } w(t)=\sum_{h=-H}^{H} \mathbf{W}_{h} \exp (i h \omega t)
$$

The equation $\dot{v}(t)=w(t) \dot{u}(t)$ becomes :

$$
\sum_{h=-H}^{H} i h \omega \mathbf{V}_{h} \exp (i h \omega t)=\left(\sum_{h=-H}^{H} \mathbf{W}_{h} \exp (i h \omega t)\right)\left(\sum_{h=-H}^{H} i h \omega \mathbf{U}_{h} \exp (i h \omega t)\right)
$$

which can be written

$$
\sum_{h=-H}^{H} i h \omega \mathbf{V}_{h} \exp (i h \omega t)=\sum_{h=-2 H}^{2 H}\left(\sum_{\substack{k_{1}+k_{2}=h \\-H \leq k_{1}, k_{2} \leq H}} \mathbf{W}_{k_{1}} i k_{2} \omega \mathbf{U}_{k_{2}}\right) \exp (i h \omega t) .
$$

The right-hand-side series is truncated at order $H$ and the harmonics are balanced :

$$
\forall h \in \llbracket-H, H \rrbracket, \quad i h \omega \mathbf{V}_{h}=\sum_{\substack{k_{1}+k_{2}=h \\-H \leq k_{1}, k_{2} \leq H}} \mathbf{W}_{k_{1}} i k_{2} \omega \mathbf{U}_{k_{2}}
$$

$\omega$ is the angular frequency of the periodic solution and thus remains positive. It can be simplified to obtain

$$
\forall h \in \llbracket-H, H \rrbracket, \quad h \mathbf{V}_{h}=\sum_{\substack{k_{1}+k_{2}=h \\-H \leq k_{1}, k_{2} \leq H}} \mathbf{W}_{k_{1}} k_{2} \mathbf{U}_{k_{2}} .
$$

This expression is quadratic with respect to the Fourier coefficients. Hence the transcendental variable $v$ can be written with a quadratic formalism in the frequency domain in an automated way. The operators $\mathbf{V} \mapsto \mathrm{d} \mathbf{L}(\mathbf{V})$ and $(\mathbf{U}, \mathbf{W}) \mapsto \mathrm{d} \mathbf{Q}(\mathbf{W}, \mathbf{U})$ are respectively the left hand side and the right hand side of the equations (40). $\mathbf{U}, \mathbf{V}$ and $\mathbf{W}$ are the vectors of Fourier coefficients of $u, v$ and $w$ respectively. It is easy to see that $\mathrm{d} \mathbf{L}$ and $\mathrm{d} \mathbf{Q}$ are respectively linear and bilinear in their argument(s).

\section{B Time-integration of the pendulum with a class of Euler schemes}

The ODE equations of the pendulum in polar coordinates (20)

$$
\left\{\begin{array}{l}
\dot{\theta}=\phi \\
\dot{\phi}=-\sin (\theta)
\end{array}\right.
$$

6 If this is not the case, an auxiliary variable $z(t)=\dot{w}(t)$ is added and the procedure explained here is applied recursively. 

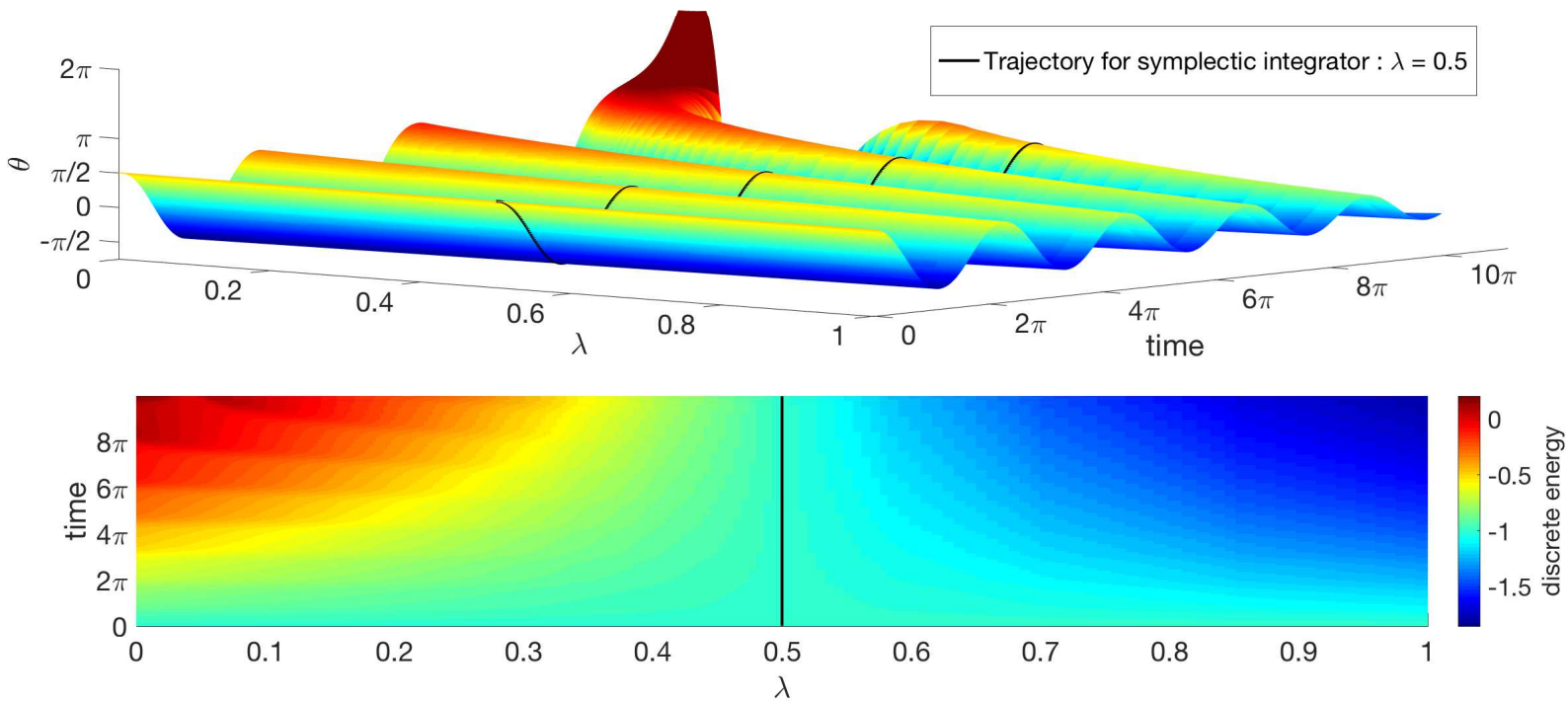

Fig. 9 The top figure shows a waterfall of the trajectories of (42). The bottom figure shows the evolution of the discrete energy (in color) with respect to time (y-axis) for all the values of $\lambda$ (x-axis). From $\lambda=0$ to $\lambda=1$, the scheme evolves from an explicit Euler to an implicit Euler. The value $\lambda=0.5$ is plotted in black to enhance the trajectory obtained with this scheme, which is known to be symplectic [32]. It is clear in the bottom figure that the scheme either creates or dissipates energy when $\lambda$ is respectively smaller or greater than 0.5 .

are solved using a generalized Euler scheme on the discretized grid $\{0, h, 2 h, \ldots,(N-1) h\}$. The standard notation $\theta_{n}=\theta(n h)$ and $\phi_{n}=\phi(n h)$ is used. The initial conditions are $\theta_{0}=\frac{\pi}{2}$ and $\phi_{0}=0$. The discretized system is then

$$
\left\{\begin{array}{l}
\theta_{n+1}=\theta_{n}+h\left((1-\lambda) \phi_{n}+\lambda \phi_{n+1}\right) \\
\phi_{n+1}=\phi_{n}-h \sin \left((1-\lambda) \theta_{n}+\lambda \theta_{n+1}\right)
\end{array}\right.
$$

where the parameter $\lambda$ is the continuation parameter and interpolates an explicit Euler scheme (for $\lambda=0)$ and an implicit Euler scheme (for $\lambda=1)$. The centered Euler scheme $\left(\lambda=\frac{1}{2}\right)$ is known to be a simple symplectic scheme [32]. It is then well suited to solve these conservative equations. The auxiliary variables $\vartheta_{n}=(1-\lambda) \theta_{n}+\lambda \theta_{n+1}$ and $F_{n}=\sin \left(\vartheta_{n}\right)$ are added to the system $(42)$ with the companion variables $G_{n}=\cos \left(\vartheta_{n}\right)$. The final quadratic recast with the differentiated forms of the auxiliary variables is

$$
\forall n \in \llbracket 0, N-1 \rrbracket,\left\{\begin{aligned}
\theta_{n+1} & =\theta_{n}+h\left((1-\lambda) \phi_{n}+\lambda \phi_{n+1}\right) & & \\
\phi_{n+1} & =\phi_{n}-h F_{n} & & \\
\vartheta_{n} & =(1-\lambda) \theta_{n}+\lambda \theta_{n+1} & & \text { and } \mathrm{d} F_{n}=G_{n} \mathrm{~d} \vartheta_{n} \\
F_{n} & =\sin \left(\vartheta_{n}\right) & & \text { and } \mathrm{d} G_{n}=-F_{n} \mathrm{~d} \vartheta_{n}
\end{aligned}\right.
$$

The top figure 9 shows the trajectories obtained for $N=500$ and $h=\frac{2 \pi}{100}$, that is over around 5 periods of the analytic solution. The bottom figure 9 shows the discrete energy $H_{n}=\frac{\phi_{n}^{2}}{2}-1-\cos \left(\theta_{n}\right)$ in color for $0 \leq \lambda \leq 1$. It is clear that energy is created when $\lambda<0.5$ while energy is dissipated when $\lambda>0.5$. The trajectory obtained for $\lambda=0.5$ is represented in black showing the stability of the energy over time, as expected for this symplectic scheme.

\section{Computation of Taylor series coefficients}

Some examples (taken from the section 1.1) of practical computation of the Taylor series coefficients are shown. However, this procedure is completely automatized from the quadratic formulation of the system of equations. It is detailed here for pedagogical purposes. The following computations illustrates how easy and straightforward it is to derive Taylor series coefficients when a quadratic recast is given.

\section{C.1 Rational case}

The system of equations is :

$$
\left\{\begin{array}{c}
y-u-x v=0 \\
u x-1=0 \\
v-x^{2}=0
\end{array}\right.
$$


Assume that $\left(x_{0}, y_{0}, u_{0}, v_{0}\right)$ is a solution of the equations. The $n$-th Taylor series coefficient of the unknown $x$ is written $x_{n}$, such that :

$$
x(a)=\sum_{n=0}^{+\infty} x_{n} a^{n}
$$

Replacing the variables by their Taylor series in the equations gives :

$$
\left\{\begin{array}{cl}
\sum_{n=0}^{+\infty} y_{n} a^{n}-\sum_{n=0}^{+\infty} u_{n} a^{n}-\left(\sum_{n=0}^{+\infty} x_{n} a^{n}\right)\left(\sum_{n=0}^{+\infty} v_{n} a^{n}\right) & =0 \\
\left(\sum_{n=0}^{+\infty} u_{n} a^{n}\right)\left(\sum_{n=0}^{+\infty} x_{n} a^{n}\right)-1 & =0 \\
\sum_{n=0}^{+\infty} v_{n} a^{n}-\left(\sum_{n=0}^{+\infty} x_{n} a^{n}\right)\left(\sum_{n=0}^{+\infty} x_{n} a^{n}\right) & =0
\end{array}\right.
$$

Developing the products and equating the terms of same order in $a$ finally gives, for $n \geq 0$ :

$$
\left\{\begin{array}{c}
y_{n}-u_{n}-\sum_{i=0}^{n} x_{i} v_{n-i}=0 \\
\sum_{i=0}^{n} u_{i} x_{n-i}-1 \delta_{n=0}=0 \\
v_{n}-\sum_{i=0}^{n} x_{i} x_{n-i}=0
\end{array}\right.
$$

where $\delta_{n=0}$ is the Kronecker symbol, its value is 1 if and only if $n=0$.

This system for $n=0$ gives

$$
\left\{\begin{array}{c}
y_{0}-u_{0}-x_{0} v_{0}=0 \\
u_{0} x_{0}-1=0 \\
v_{0}-x_{0}^{2}=0
\end{array}\right.
$$

which is verified from the assumption that $\left(x_{0}, y_{0}, u_{0}, v_{0}\right)$ is a solution of the equations (44).

For $n \geq 1$, the terms of higher order are put apart to make the recurrence relations appear :

$$
\left\{\begin{array}{cl}
y_{n}-u_{n}-x_{0} v_{n}-x_{n} v_{0} & =\sum_{i=1}^{n-1} x_{i} v_{n-i} \\
u_{0} x_{n}+u_{n} x_{0} & =-\sum_{i=1}^{n-1} u_{i} x_{n-i} \\
v_{n}-2 x_{0} x_{n} & =\sum_{i=1}^{n-1} x_{i} x_{n-i}
\end{array}\right.
$$

which is equivalent to the linear system

$$
\mathbf{J}_{0} \times\left[\begin{array}{l}
x_{n} \\
y_{n} \\
u_{n} \\
v_{n}
\end{array}\right]=\left[\begin{array}{c}
\sum_{i=1}^{n-1} x_{i} v_{n-i} \\
-\sum_{i=1}^{n}-1 \\
\sum_{i=1}^{n=1} x_{i} x_{n-i} x_{n-i}
\end{array}\right]
$$

where $\mathbf{J}_{0}=\left[\begin{array}{cccc}-v_{0} & 1 & -1 & x_{0} \\ u_{0} & 0 & x_{0} & 0 \\ -2 x_{0} & 0 & 0 & 1\end{array}\right]$ is the Jacobian matrix of the system at the solution point $\left(x_{0}, y_{0}, u_{0}, v_{0}\right)$. From the knowledge of the initial solution point $\left(x_{0}, y_{0}, u_{0}, v_{0}\right)$ it is then possible to deduce the whole Taylor series of the variables $x, y, u$ and $v$.

\section{C.2 Transcendental case}

The system of equations and its differential form are given by :

$$
\begin{cases}y-\sin (x)=0 \text { and } & \mathrm{d} y-z \mathrm{~d} x=0 \\ z-\cos (x)=0 \text { and } & \mathrm{d} z+y \mathrm{~d} x=0\end{cases}
$$

Assume that $\left(x_{0}, y_{0}, z_{0}\right)$ is a solution of the equations. Thus $y_{0}=\sin \left(x_{0}\right)$ and $z_{0}=\cos \left(x_{0}\right)$. Contrarily to the previous section, the differential form of the equations is used to compute the higher order coefficients of the Taylor series by writing

$$
\left\{\begin{array}{l}
\frac{\partial y}{\partial a}-z(a) \frac{\partial x}{\partial a}=0 \\
\frac{\partial z}{\partial a}+y(a) \frac{\partial x}{\partial a}=0
\end{array}\right.
$$

The derivative $\frac{\partial x}{\partial a}$ of the Taylor series of the unknown $x$ with respect to $a$ is :

$$
\frac{\partial x}{\partial a}(a)=\sum_{n=0}^{+\infty}(n+1) x_{n+1} a^{n}
$$

Replacing the variables by the corresponding series in the equations (52) gives :

$$
\left\{\begin{array}{l}
\sum_{n=0}^{+\infty}(n+1) y_{n+1} a^{n}-\left(\sum_{n=0}^{+\infty} z_{n} a^{n}\right)\left(\sum_{n=0}^{+\infty}(n+1) x_{n+1} a^{n}\right)=0 \\
\sum_{n=0}^{+\infty}(n+1) z_{n+1} a^{n}+\left(\sum_{n=0}^{+\infty} y_{n} a^{n}\right)\left(\sum_{n=0}^{+\infty}(n+1) x_{n+1} a^{n}\right)=0
\end{array}\right.
$$

Developing the products and equating the terms of same order in $a$ finally gives, for $n \geq 0$ :

$$
\left\{\begin{array}{l}
(n+1) y_{n+1}-\sum_{i=0}^{n} z_{i}(n+1-i) x_{n+1-i}=0 \\
(n+1) z_{n+1}+\sum_{i=0}^{n} y_{i}(n+1-i) x_{n+1-i}=0
\end{array}\right.
$$


The terms of higher order are put apart and everything is divided by $(n+1)$ to make the recurrence relations appear :

$$
\left\{\begin{array}{l}
y_{n+1}-z_{0} x_{n+1}=\sum_{i=1}^{n} z_{i} \frac{n+1-i}{n+1} x_{n+1-i} \\
z_{n+1}+y_{0} x_{n+1}=-\sum_{i=1}^{n} y_{i} \frac{n+1-i}{n+1} x_{n+1-i}
\end{array}\right.
$$

which is equivalent to the linear systems

$$
\mathbf{J}_{0} \times\left[\begin{array}{l}
x_{n+1} \\
y_{n+1} \\
z_{n+1}
\end{array}\right]=\left[\begin{array}{c}
\sum_{i=1}^{n} z_{i}(n+1-i) x_{n+1-i} \\
-\sum_{i=1}^{n} y_{i}(n+1-i) x_{n+1-i}
\end{array}\right]
$$

where $\mathbf{J}_{0}=\left[\begin{array}{ccc}-z_{0} & 1 & 0 \\ y_{0} & 0 & 1\end{array}\right]$ is the Jacobian matrix of the system at the solution point $\left(x_{0}, y_{0}, z_{0}\right)$. From the knowledge of the initial solution point $\left(x_{0}, y_{0}, z_{0}\right)$ it is then possible to deduce the whole Taylor series of the variables $x, y$ and $z$.

\section{References}

1. Barton, D.A., Krauskopf, B., Wilson, R.E.: Collocation schemes for periodic solutions of neutral delay differential equations. Journal of Difference Equations and Applications 12(11), 1087-1101 (2006)

2. Bentvelsen, B., Lazarus, A.: Modal and stability analysis of structures in periodic elastic states: application to the ziegler column. Nonlinear Dynamics 91(2), 1349-1370 (2018)

3. Beyn, W.J., Champneys, A., Doedel, E., Govaerts, W., Kuznetsov, Y.A., Sandstede, B.: Numerical continuation, and computation of normal forms. In: In Handbook of dynamical systems III: Towards applications. Citeseer (2001)

4. Boumédiène, F., Miloudi, A., Cadou, J., Duigou, L., Boutyour, E.: Nonlinear forced vibration of damped plates by an asymptotic numerical method. Computers \& Structures 87(23-24), 1508-1515 (2009)

5. Cameron, T., Griffin, J.: An alternating frequency/time domain method for calculating the steady-state response of nonlinear dynamic systems. Journal of applied mechanics 56(1), 149-154 (1989)

6. Charpentier, I., Cochelin, B.: Towards a full higher order ad-based continuation and bifurcation framework. Optimization Methods \& Software (2018)

7. Charpentier, I., Lejeune, A., Potier-Ferry, M.: The diamant approach for an efficient automatic differentiation of the asymptotic numerical method. Advances in Automatic Differentiation pp. 139-149 (2008)

8. Claude, B., Duigou, L., Girault, G., Cadou, J.M.: Eigensolutions to a vibroacoustic interior coupled problem with a perturbation method. Comptes Rendus Mécanique 345(2), 130-136 (2017)

9. Cochelin, B.: A path-following technique via an asymptotic-numerical method. Computers \& structures 53(5), 1181-1192 (1994)

10. Cochelin, B., Damil, N., Potier-Ferry, M.: Asymptotic-numerical methods and pade approximants for non-linear elastic structures. International journal for numerical methods in engineering 37(7), 1187-1213 (1994)

11. Cochelin, B., Damil, N., Potier-Ferry, M.: The asymptotic-numerical method: an efficient perturbation technique for nonlinear structural mechanics. Revue européenne des éléments finis 3(2), 281-297 (1994)

12. Cochelin, B., Vergez, C.: A high order purely frequency-based harmonic balance formulation for continuation of periodic solutions. Journal of sound and vibration 324(1), 243-262 (2009)

13. Dhooge, A., Govaerts, W., Kuznetsov, Y.A.: Matcont: a matlab package for numerical bifurcation analysis of odes. ACM Transactions on Mathematical Software (TOMS) 29(2), 141-164 (2003)

14. Doedel, E.J.: Auto: A program for the automatic bifurcation analysis of autonomous systems. Congr. Numer 30, 265-284 (1981)

15. Doedel, E.J., Govaerts, W., Kuznetsov, Y.A.: Computation of periodic solution bifurcations in odes using bordered systems. SIAM Journal on Numerical Analysis 41(2), 401-435 (2003)

16. Doedel, E.J., Heinemann, R.F.: Numerical computation of periodic solution branches and oscillatory dynamics of the stirred tank reactor with a yields b yields c reactions. Tech. rep., WISCONSIN UNIV-MADISON MATHEMATICS RESEARCH CENTER (1982)

17. Engelborghs, K., Luzyanina, T., Roose, D.: Numerical bifurcation analysis of delay differential equations using dde-biftool. ACM Transactions on Mathematical Software (TOMS) 28(1), 1-21 (2002)

18. Farner, S., Vergez, C., Kergomard, J., Lizée, A.: Contribution to harmonic balance calculations of self-sustained periodic oscillations with focus on single-reed instruments. The Journal of the Acoustical Society of America 119(3), 1794-1804 (2006)

19. Fontanela, F., Grolet, A., Salles, L., Hoffmann, N.: Computation of quasi-periodic localised vibrations in nonlinear cyclic and symmetric structures using harmonic balance methods. Journal of Sound and Vibration 438, 54-65 (2019)

20. Fritzsche, K., Grauert, H.: From holomorphic functions to complex manifolds, vol. 213. Springer Science \& Business Media (2012)

21. Griewank, A., Walther, A.: Evaluating derivatives: principles and techniques of algorithmic differentiation, vol. 105. Siam (2008)

22. Guillot, L., Cochelin, B., Vergez, C.: A generic and efficient taylor series based continuation method using a quadratic recast of smooth nonlinear systems. International Journal for Numerical Methods in Engineering 0(ja) (2019). DOI 10.1002/nme.6049. URL https://onlinelibrary.wiley.com/doi/abs/10.1002/nme.6049

23. Guillot, L., Vergez, C., Cochelin, B.: Continuation of periodic solutions of various types of Delay Differential Equations using Asymptotic Numerical Method and Harmonic Balance Method. Nonlinear Dynamics (2019). DOI 10.1007/s11071-019-04958-y

24. Guillot, L., Vigué, P., Vergez, C., Cochelin, B.: Continuation of quasi-periodic solutions with two-frequency harmonic balance method. Journal of Sound and Vibration 394, 434-450 (2017)

25. Jean, A., Nelson, H.: Periodic response investigation of large order non-linear rotordynamic systems using collocation. Journal of Sound and Vibration 143(3), 473-489 (1990)

26. Karkar, S., Cochelin, B., Vergez, C.: A comparative study of the harmonic balance method and the orthogonal collocation method on stiff nonlinear systems. Journal of Sound and Vibration 333(12), 2554-2567 (2014) 
27. Keller, H.: Lectures on numerical methods in bifurcation problems. Applied Mathematics 217, 50 (1987)

28. Khibnik, A.I., Kuznetsov, Y.A., Levitin, V.V., Nikolaev, E.V.: Continuation techniques and interactive software for bifurcation analysis of odes and iterated maps. Physica D: Nonlinear Phenomena 62(1-4), 360-371 (1993)

29. Krylov, N.M., Bogoliubov, N.N.: Introduction to non-linear mechanics. Princeton university press (1949 (English translation of Russian edition from 1936)

30. marquis de Laplace, P.S.: Traité de mécanique céleste, vol. 1. Crapelet (1799)

31. Lazarus, A., Thomas, O.: A harmonic-based method for computing the stability of periodic solutions of dynamical systems. Comptes Rendus Mécanique 338(9), 510-517 (2010)

32. Leimkuhler, B., Reich, S.: Simulating hamiltonian dynamics, vol. 14. Cambridge university press (2004)

33. Lévy, L.: Précis élémentaire de la théorie des fonctions elliptiques avec tables numériques et applications. Gauthier-Villars (1898)

34. Liao, S.: Beyond perturbation: introduction to the homotopy analysis method. Chapman and Hall/CRC (2003)

35. Munoz-Almaraz, F.J., Freire, E., Galán, J., Doedel, E., Vanderbauwhede, A.: Continuation of periodic orbits in conservative and hamiltonian systems. Physica D: Nonlinear Phenomena 181(1-2), 1-38 (2003)

36. Nakhla, M., Vlach, J.: A piecewise harmonic balance technique for determination of periodic response of nonlinear systems. IEEE Transactions on Circuits and Systems 23(2), 85-91 (1976)

37. Newmark, N.M.: A method of computation for structural dynamics. Journal of the engineering mechanics division 85(3), 67-94 (1959)

38. Peeters, M., Viguié, R., Sérandour, G., Kerschen, G., Golinval, J.C.: Nonlinear normal modes, part ii: Toward a practical computation using numerical continuation techniques. Mechanical systems and signal processing 23(1), 195-216 (2009)

39. Schilder, F., Osinga, H.M., Vogt, W.: Continuation of quasi-periodic invariant tori. SIAM Journal on Applied Dynamical Systems 4(3), 459-488 (2005)

40. Seydel, R.: From equilibrium to chaos: practical bifurcation and stability analysis. North-Holland (1988)

41. Vigué, P.: Solutions périodiques et quasi-périodiques de systèmes dynamiques d'ordre entier ou fractionnaire : applications à la corde frottée. Ph.D. thesis (2017). URL http://www.theses.fr/2017AIXM0306. Thèse de doctorat dirigée par Vergez, Christophe et Cochelin, Bruno Sciences de l'ingénieur. Acoustique Aix-Marseille 2017

42. Vigué, P., Vergez, C., Lombard, B., Cochelin, B.: Continuation of periodic solutions for systems with fractional derivatives. Nonlinear Dynamics pp. 1-15 (2018)

43. Zhou, B., Thouverez, F., Lenoir, D.: A variable-coefficient harmonic balance method for the prediction of quasi-periodic response in nonlinear systems. Mechanical Systems and Signal Processing 64, 233-244 (2015) 